\title{
Systemic Lupus Erythematosus (SLE) - A Review of clinical approach for diagnosis
} and current treatment strategies

\section{Narani $A^{1}$ \\ ${ }^{1}$ Teaching Hospital Jaffna}

\section{Abstract}

Systemic lupus erythematosus (SLE) is a chronic, complicated and challenging disease to diagnose and treat. The etiology of SLE is unknown and various criteria were created to aide in the diagnosis, focusing on clinical manifestations and antibody profiles specific to SLE. Treatment options are limited to a few medications to control the inflammation and decrease organ damage. Treatment strategies vary according to the specific organ complication.

Keywords: Systemic Lupus Erythematosus, Auto immunity, Immune complex, Lupus nephritis

\section{SLE Introduction}

Systemic lupus erythematosus (SLE) is a chronic autoimmune disease that can affect almost any organ system such as the skin, joints, kidneys, nervous system, heart, lungs and the serous membranes. Its presentation and course are highly variable, ranging from indolent to fulminant. (1)

\section{SLE Epidemiology}

The frequency of SLE varies by sex, age, race and ethnicity. More than $90 \%$ of cases of SLE occur in women, frequently starting at childbearing age. The risk of SLE development in men is similar to that of pre pubertal or postmenopausal women. The female to male ratio peaks at 11:1 during the childbearing years. Onset of SLE is usually after puberty, typically in 20s and 30s.

\section{SLE Pathogenesis}

The etiology of SLE is unknown. Certain risk factors have been identified and shown to contribute to disease susceptibility or activate the immune system causing an inflammatory response, ultimately leading to the development of the disease. Predisposition to SLE is influenced by genetic factors. The female predominance in SLE may be explained, in part, by the contribution of certain hormones. Environmental factors, such as smoking, exposure to ultraviolet light, viral infections, and specific medications (e.g. sulfonamide antibiotics) are known to trigger SLE. The pathogenesis of SLE is complex with contribution from many components of the immune system. With the underlying genetic predisposition and in response to various triggers the balance of the immune system shifts towards reacting against itself, rather than self-tolerance. T and B cells become activated, leading to antibody production and eventual immune complex formation. These complexes circulate and deposit in critical tissues causing organ injury.

\section{SLE Diagnosis}

Classification criteria have been derived for SLE, mainly for research purposes, to achieve population homogeneity among research studies. The American College of Rheumatology (ACR) published criteria in 1982, which were revised in 1997. The Systemic Lupus Collaborating Clinics (SLICC) international group undertook the evaluation and further revision of the above criteria resulting in a new classification system that is based on clinical and immunologic manifestations. In an actual clinical practice setting, both criteria were analyzed; it was determined that the SLICC 2012 criteria were more sensitive and may allow patients to be classified with SLE earlier in the disease course. In the clinical setting, these criteria can be used as an aid in diagnosis, but formal diagnostic criteria for SLE are lacking.

The SLICC criteria have also been criticized because they were derived comparing the expert's 
decision ("gold standard") with a standardized group of manifestations. Moreover, as SLICC criteria emphasize immunological and hematological events, it might be possible that subjects classified through SLICC criteria may exhibit less clinically significant multisystem involvement compared with subjects classified through ACR criteria. (2)

In 2017, the EULAR and ACR joined in a four-phase project to develop more sensitive (especially for initial classification) and more specific SLE classification criteria. (3)

\section{New ACR and EULAR criteria for classification of SLE}

All patients classified as having systemic lupus erythematous must have a serum titer of antinuclear antibody of at least 1:80 on human epithelial-2-positive cells or an equivalent positive test .In addition, a patient must tally at least 10 points from these criteria. A criterion is not counted if it has a more likely explanation than SLE. Occurrence of the criterion only once is sufficient to tally the relevant points, and the time when a patient is positive for other criteria.SLE classification requires points from at least one clinical domain, and if a patient is positive for more than one criterion in a domain only the criterion with the highest point value counts:

\begin{tabular}{|c|c|c|c|}
\hline Clinical domains & Points & Immunological domains & Points \\
\hline Constitutional domain & \multirow{2}{*}{2} & Antiphospholipid antibody & \\
\hline Fever & & domain & \\
\hline Cutaneous domain & & Anticardiolipin $1 \mathrm{gG}>40 \mathrm{GPL}$ or & 2 \\
\hline Non scarring alopecia & 2 & anti $\beta 2 \mathrm{GP} 1 \mathrm{IgG}>40$ units or & \\
\hline Oral Ulcers & 2 & lupus anticoagulant & \\
\hline Subacute cutaneous or discoid lupus & 4 & Complement proteins domain & \\
\hline Acute cutaneous lupus & 6 & Low $\mathrm{C} 3$ or low $\mathrm{C} 4$ & 3 \\
\hline Arthritis domain & & Low $\mathrm{C} 3$ and low $\mathrm{C} 4$ & 4 \\
\hline Synovitis in at least two joints & 6 & Highly specific antibodies domain & \\
\hline or tenderness in at least two & & Anti- ds DNA antibody & 6 \\
\hline joints, and at least $30 \mathrm{~min}$ of & & Anti-Smith antibody & 6 \\
\hline \multicolumn{2}{|l|}{ morning stiffness } & & \\
\hline \multicolumn{2}{|l|}{ Neurologic domain } & & \\
\hline Derillium & 2 & & \\
\hline Psychosis & 3 & & \\
\hline Seizure & 5 & & \\
\hline \multicolumn{2}{|l|}{ Sirositis domain } & & \\
\hline Pleural or pericardial effusion & 5 & & \\
\hline Acute Pericarditis & 6 & & \\
\hline \multicolumn{2}{|l|}{ Hematologic domain } & & \\
\hline Leukopenia & 3 & & \\
\hline Thrombocytopenia & 4 & & \\
\hline Autoimmune hemolysis & 4 & & \\
\hline \multicolumn{2}{|l|}{ Renal domain } & & \\
\hline Proteinuria $>0.5 \mathrm{~g} / 24 \mathrm{hr}$ & 4 & & \\
\hline Class 11 or V lupus nephritis & 8 & & \\
\hline Class 111 or V lupus nephritis & 10 & & \\
\hline
\end{tabular}

Source: Dr.Johnson 


\section{Treatment of SLE}

\section{Overarching principles}

SLE represents a challenge for the treating physician in terms of diagnosis and treatment. Its protean manifestations, often multisystem but occasionally limited to a few or single organ, have led some physicians to focus exclusively on evidence of serological autoimmunity (antinuclear and more specific auto antibodies), for a disease where diagnosis is clinical after excluding competing diagnoses. Monitoring of SLE through validated disease activity and chronicity indices, including physician global assessment (PGA), is recommended. For patients with severe disease, multidisciplinary care in dedicated lupus centers is desirable. Immunosuppressive (IS) therapy (for induction and maintenance of remission) is indicated in organ-threatening lupus. (4)

\section{Recommendations Goals of treatment}

To improve long-term patient outcomes, management should aim at remission of disease symptoms and signs, prevention of damage accrual and minimization of drug side-effects, as well as improvement of quality of life.

Complete remission (absence of clinical activity with no use of GC and IS drugs) is infrequent. (5) To this end, newly defined low disease activity states (based on a SLEDAI score $\leq 3$ on anti malarial, or alternatively SLEDAI $\leq 4, \mathrm{PGA} \leq 1$ with $\mathrm{GC} \leq 7.5$ $\mathrm{mg}$ of prednisone and well tolerated IS agents) have shown comparable rates with remission, regarding halting of damage accrual (OR 0.5-0.7 for increase in damage index) and prevention of flares.

Accordingly, treatment in SLE should aim at remission or, if this state cannot be achieved, at low disease activity in all organ systems. (6) In LN, therapy should aim at least partial remission (defined as $\geq 50 \%$ reduction in proteinuria [UPr] to subnephrotic levels and serum creatinine within $10 \%$ from baseline) by $6-12$ months; complete renal remission (proteinuria $<500 \mathrm{mg} / 24$ hours and SCr within $10 \%$ from baseline), however, may require longer treatment duration, often more than 12 and until 24 months. In monitoring renal response, reduction of UPr (to less than $0.8 \mathrm{~g} /$ day) following treatment is more important than residual haematuria. Patients with more severe proteinuria and longer-standing disease are less likely to respond or show more delayed responses.

Prevention of disease flares is an additional milestone of SLE treatment. Flares are common in the disease course and contribute significantly to organ damage accrual and worse outcome. Consistently reported risk factors for a higher disease flare rate include younger age at disease onset, no use of anti malarial drugs, persistent generalized disease activity and serological activity (anti-dsDNA, low complement). Assessment of adherence to drug treatment, close monitoring and optimization of disease control in these patients may reduce the risk for a flare. (7)

\section{DRUGS USED IN SLE \\ Hydroxychloroquine}

Hydroxychloroquine (HCQ) is recommended for all patients with SLE. There is evidence for multiple beneficial effects of HCQ in SLE, yet poor adherence to treatment is not uncommon.

Concerns for retinal toxicity with long-term HCQ therapy led to the use of more sensitive screening techniques, with a prevalence of retinal abnormalities exceeding $10 \%$ after 20 years of continuous use. (8) Major risk factors for retinopathy include duration of treatment (OR 4.71 for every 5 years of use), dose (OR 3.34 for every $100 \mathrm{mg}$ daily dose), chronic kidney disease (adjusted OR 8.56) and pre-existing retinal or macular disease. Based on existing evidence suggesting that the risk of toxicity is very low for doses below $5 \mathrm{mg} / \mathrm{kg}$ real body weight, the daily dose should not exceed this threshold.

Patients in long-standing remission may have their dose lowered, although no studies have formally addressed this strategy. The choice of quinacrine, an alternative anti malarial, can be considered in patients with cutaneous manifestations and HCQinduced retinal toxicity. (9) 


\section{Glucocorticoids}

GC can provide rapid symptom relief, but the medium to long-term aim should be to minimize daily dose to $\leq 7.5 \mathrm{mg} /$ day prednisone equivalent or to discontinue them, because long-term GC therapy can have various detrimental effects including irreversible organ damage. (10) Risks are substantially increased at continuous GC doses above $7.5 \mathrm{mg} /$ day, with some studies suggesting that also lower doses might be harmful.

To this end, two approaches can be considered: (1) use of pulses of intravenous methylprednisolone (MP) of various doses (depending on severity and body weight), which take advantage of the rapid non-genomic effects of GC and may allow for a lower starting dose and faster tapering of PO $\mathrm{GC}$, and early initiation of IS agents, to facilitate tapering and eventual discontinuation of oral GC. High-dose intravenous MP (usually 250-1000 $\mathrm{mg}$ /day for 3 days) is often used in acute, organthreatening disease (renal, neuropsychiatric) after excluding infections. (11)

\section{Immunosuppressive (IS) drugs}

Consequent initiation of IS drugs facilitates a more rapid GC tapering and may prevent disease flares. The choice of agent depends on prevailing disease manifestation(s), patient age and childbearing potential, safety concerns and cost. (12)

Methotrexate (MTX) and azathioprine (AZA) should be considered in patients with poor symptom control after a trial with GC and HCQ or when HCQ alone is unlikely to be sufficient, due to the large experience gained with their use and their relatively safe profile. (12) Mycophenolate mofetil (MMF) is a potent immunosuppressant with efficacy in renal and non-renal lupus (although not in neuropsychiatric disease).

In a recent randomized, open-label trial in extra renal SLE, enteric-coated mycophenolate sodium (EC-MPS) was superior to AZA in achieving remission and reducing flares. However, it's teratogenic potential (needs to be discontinued at least 6 weeks before conceiving), along with its higher cost compared with AZA or MTX, and poses a limitation towards universal recommendation in women of reproductive age with non-renal manifestations. Cyclophosphamide (CYC) can be considered in organ-threatening disease (especially renal, cardiopulmonary or neuropsychiatric) and only as rescue therapy in refractory non-major organ manifestations; due to its gonado-toxic effects, it should be used with caution in women and men of fertile age. (13)

\section{Biological agents}

There is evidence to support beneficial effects of B-cell targeting agents in SL. Belimumab should be considered in extra renal disease with inadequate control (ongoing disease activity or frequent flares) to first-line treatments (typically including combination of HCQ and prednisone with or without IS agents), and inability to taper GC daily dose to acceptable levels (ie, maximum $7.5 \mathrm{mg} /$ day). (14)

Patients with persistent disease may benefit from belimumab; more likely to respond are patients with high disease activity (eg, SLEDAI $>10$ ), prednisone dose $>7.5 \mathrm{mg} /$ day and serological activity (low $\mathrm{C} 3 / \mathrm{C} 4$, high anti-dsDNA titres), with cutaneous, musculoskeletal and serological manifestations responding the most. (15)

Due to the negative results of randomized controlled trials (RCTs), RTX is currently only used off-label, in patients with severe renal or extra renal (mainly haematological and neuropsychiatric) disease refractory to other IS agents and/or belimumab, or in patients with contraindications to these drugs.

\section{CONCLUSION}

SLE remains a challenging disorder that requires an interdisciplinary approach with a team of healthcare providers to diagnose, manage and tailor treatment to individual patient needs.

\section{References}

1. Cojocaru, M. et al. 'Manifestations of systemic lupus erythematosus', Maedica. Media Med Publicis, 6(4), pp. 330-336, 2011. 
2. Pons-Estel, G. J. et al. 'The American College of Rheumatology and the Systemic Lupus International Collaborating Clinics classification criteria for systemic lupus erythematosus in two multiethnic cohorts: a commentary', Lupus. Sage Publications Sage UK: London, England, 23(1), pp. 3-9,2014.

3. Petri, M. et al. 'Validation of Proposed EULAR / Acr SLE Classi cation Criteria Versus SLICC SLE Classi cation Criteria',2012.

4. Ward, M. M. 'Hospital experience and mortality in patients with systemic lupus erythematosus', Arthritis \& Rheumatism: Official Journal of the American College of Rheumatology. Wiley Online Library, 42(5), pp. 891-898,1999.

5. Medina-Quiñones, C. V et al. 'Analysis of Complete Remission in Systemic Lupus Erythematosus Patients Over a 32-Year Period', Arthritis care \& research. Wiley Online Library, 68(7), pp. 981-987,2016.

6. Ugarte-Gil, M.F. et al. 'Remission and Low Disease Activity Status (LDAS) protect lupus patients from damage occurrence: data from a multiethnic, multinational Latin American Lupus Cohort (GLADEL)', Annals of the rheumatic diseases. BMJ Publishing Group Ltd, 76(12), pp. 20712074,2017.

7. Touma, Z. et al. 'Time to recovery from proteinuria in patients with lupus nephritis receiving standard treatment', The Journal of rheumatology. The Journal of Rheumatology, 41(4), pp. 688-697,2014.

8. Costedoat-Chalumeau, N. et al. 'Very low blood hydroxychloroquine concentration as an objective marker of poor adherence to treatment of systemic lupus erythematosus', Annals of the rheumatic diseases. BMJ Publishing Group Ltd, 66(6), pp. 821824,2007 ..
9. Kim, J.-W. et al. 'Risk of retinal toxicity in longterm users of hydroxychloroquine', The Journal of rheumatology. The Journal of Rheumatology, 44(11), pp. 1674$1679,2017$.

10. Lim, L. S. H. et al. 'From Childhood to Adulthood: The Trajectory of Damage in Patients With Juvenile-Onset Systemic Lupus Erythematosus', Arthritis care \& research. Wiley Online Library, 69(11), pp. 1627-1635,2017.

11. Pego-Reigosa, J. M. et al. 'Efficacy and safety of nonbiologic immunosuppressants in the treatment of nonrenal systemic lupus erythematosus: a systematic review', Arthritis care \& research. Wiley Online Library, 65(11), pp. 1775-1785,2013.

12. Sakthiswary, R. and Suresh, E. 'Methotrexate in systemic lupus erythematosus: a systematic review of its efficacy', Lupus. Sage Publications Sage UK: London, England, 23(3), pp. 225-235,2014.

13. Bernatsky, S. et al. 'Lymphoma risk in systemic lupus: effects of disease activity versus treatment', Annals of the rheumatic diseases. BMJ Publishing Group Ltd, 73(1), pp. 138-142,2014.

14. Navarra, S. V et al.'Efficacy and safety of belimumab in patients with active systemic lupus erythematosus: a randomised, placebo-controlled, phase 3 trial', The Lancet. Elsevier, 377(9767), pp. 721731,2011 .

15. Iaccarino, L. et al. 'Clinical predictors of response and discontinuation of belimumab in patients with systemic lupus erythematosus in real life setting. Results of a large, multicentric, nationwide study', Journal of autoimmunity. Elsevier, 86, pp. $1-8,2018$. 